Textures and Microstructures, 1987, Vol. 7, pp. 149-170

Photocopying permitted by license only

(C) 1987 Gordon and Breach Science Publishers Inc.

Printed in the United Kingdom

\title{
Development of Deformation Textures in Polycrystalline Copper Experiments and Model Predictions
}

\author{
H. NAAMAN, ${ }^{*}$ R. TALREJA,* D. JUUL JENSEN $\ddagger$ \\ and N. HANSEN $\ddagger$ \\ * Solid Mechanics Department, Technical University of Denmark, DK-2800 \\ Lyngby, Denmark \\ ‡ Metallurgy Department, Risø National Laboratory, DK-4000 Roskilde, \\ Denmark
}

(Received 11 June 1986)

The textural development and flow stress have been determined in compression and tension of copper (99.999\%). For strains below 1.4 (compression) and 1.0 (tension) the textural development is in qualitative agreement with Taylor-model predictions, i.e. a maximum concentration at the $\langle 110\rangle$ and $\langle 111\rangle+\langle 100\rangle$ orientations for compression and tension, respectively. The grain size (23 and $125 \mu \mathrm{m})$ has only a relatively small effect on the textural development. For large strains 1.4-2.9 (compression) the textural development is in broad agreement with relaxedconstraints (RC) model predictions. In the strain range where the Taylor-model is prevalent the textural development has only a small effect on the $M$-factor, i.e. on the flow stress-strain relationship.

KEY WORDS: Textural development, flow stress, Taylor-models.

\section{INTRODUCTION}

The textural development during plastic deformation of polycrystalline materials gives important information about the deformation mechanisms and the flow stress behaviour. Deformation textures have been predicted on the basis of a number of theoretical approaches (Chin et al., 1967, Leffers, 1968, Kallend and Davies, 
1972, Honeff and Meckifíg, 1978, Aernoudt, 1978, Kocks and Canova, 1981) which are important for an understanding of the textural development. However, such models must be verified by experimental studies of real materials.

In the present study the textures of polycrystalline copper deformed in compression and in tension have been compared with model predictions for fcc materials. Two grain sizes have been studied and the textural development as well as the flow stress has been measured.

\section{EXPERIMENTAL TECHNIQUES}

\section{A Specimen preparation}

The material used was pure copper $(99.999 \%)$ cold drawn and recrystallized at $400^{\circ} \mathrm{C}$ for one hour. In order to obtain specimens with a larger grain size, a heat treatment was carried out as shown in Table 1.

\section{B Deformation in compression}

The true flow stress was determined as a function of the true plastic strain using a strain rate of $10^{-3} \mathrm{~s}^{-1}$. The strain rate was kept almost constant by a stepwise regulation of the loading speed. The initial height $\left(h_{0}\right)$ of the specimens was $6.6 \mathrm{~mm}$ and the height to diameter ratio was 1.5 . The height $(h)$ at a given strain was measured on basis of the cross-head movement taking into consideration the elastic deformation of the frame. The true plastic strain $(e)$ was calculated as $\ln \left(h_{0} / h\right)$.

To reduce the friction between the specimens and tools, the specimen ends were lubricated and thin Teflon foils were positioned on the tools. By this technique the shape of the specimen could be

Table 1 Sample code and grain sizes

\begin{tabular}{llc}
\hline Sample code & Heat treatment & Mean grain diameter, $\mu \mathrm{m}$ \\
\hline$A$ & As recrystallized & 23 \\
$B$ & As $A+48 h$ at $450^{\circ} \mathrm{C}$ & 125 \\
\hline
\end{tabular}


kept almost cylindrical to a strain of approximately 1.4. (The $B$ specimens showed a slight tendency to deform into an elliptical shape at large strains).

Compression to larger strains than 1.4 was carried out on a few $A$ specimens by a technique applied by Taylor and Quinney (1934). In order to minimize the friction effects, the diameter of the specimen was reduced by machining after a certain strain, whereafter the compression was continued. By this technique specimens were compressed to a maximum strain of 2.9. The specimen geometry at these large strains was not well-defined, and therefore the flow stress could not be determined with sufficient accuracy. As concerns the texture measurements more experimental scatter at large strain must be expected, thus volume concentrations of individual textures components have only been calculated for strains below 1.4.

\section{Deformation in tension}

The true flow stress was determined as a function of the true plastic strain using a strain rate of $10^{-3} \mathrm{~s}^{-1}$. The test specimens were of two types:

a) Cylinder specimens with a diameter of $4.47 \mathrm{~mm}$ which could be strained to a true strain of about 0.3 before the start of necking. At strains below 0.2 the elongation was measured directly by an extensometer whereas at larger strains the elongation was determined on the basis of the cross-head movement.

b) Hour glass-shaped specimens (Tome et al. 1984) with a minimum diameter of $4.2 \mathrm{~mm}$ which were strained to about 1.0 . (For this type of specimens, a large strain can be achieved, as the shape of the specimen forces the strain localization (necking) to occur at the minimum cross section). The diameter of the specimens was determined by a sliding caliper and the experimental data were corrected according to the method of Bridgman (1952) to allow for the flow stress increase caused by triaxiality in the deformation zone.

\section{Texture measurements}

The texture measurements were carried out by neutron diffraction, and the texture data is presented as inverse pole figures derived 

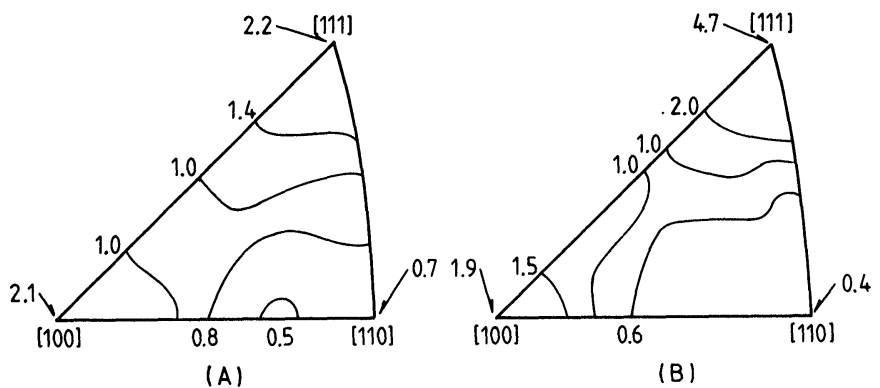

Figure 1 Inverse pole figures for $A$ and $B$ specimens in the undeformed state.

from three-dimensional orientation distribution functions (ODF's). In the present work the inverse pole figures were determined on the basis of three quarter pole figures $(111,200,220)$. Details about the experimental techniques as well as of the texture calculations are given elsewhere (Juul Jensen and Kjems 1983, Juul Jensen et al. 1984). The volume fraction of specific texture components is normally calculated by integration over 15 or $20^{\circ}$. Examples of inverse pole figures are shown in Figure 1 for the undeformed materials. It is apparent that the starting texture is not random, but consists of a mixed $\langle 100\rangle$ and $\langle 111\rangle$ texture.

The specimens for texture measurements after compression were analysed without further preparation. After large degrees of deformation the very thin specimens were glued together to obtain a sufficient volume for the diffraction measurements. After tensile testing, samples $10 \mathrm{~mm}$ long were cut from the cylindrical test specimens, and in the case of hour glass specimens samples $1.5 \mathrm{~mm}$ high were cut from the position of minimum area and three of such discs were glued together. (The latter preparation technique was applied in order to minimize the effect of a (relatively small) non-uniform distribution of the strain along the length of the hour glass specimens).

\section{EXPERIMENTAL RESULTS}

\section{A Flow stress}

The true stress-true strain curves in compression and in tension are shown in Figures 2 and 3, respectively. These results are in good 


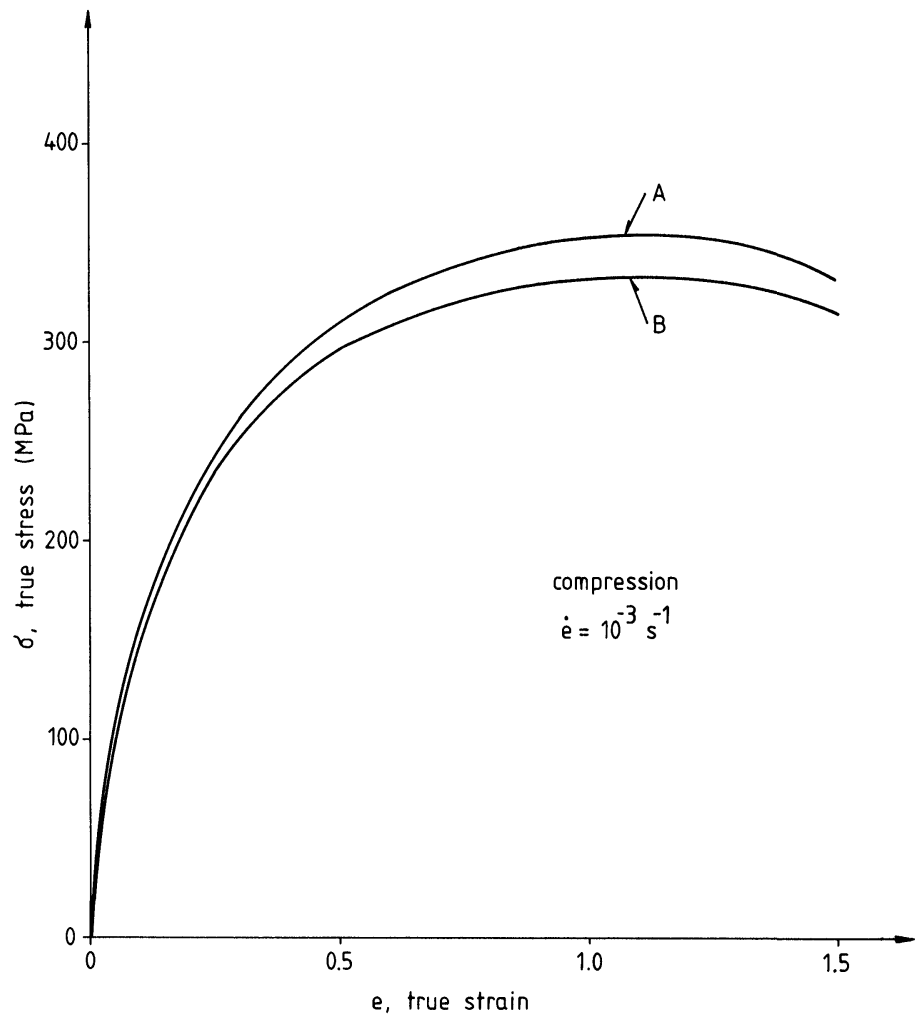

Figure 2 True stress-true strain curves in compression.

agreement with those obtained by Tome et al. (1984) for OFHC copper $(99.95 \%)$ with a grain size of approximately $20 \mu \mathrm{m}$. The present flow stress data (for $A$ specimens) is approximately 5\% lower than those obtained by Tome et al. (1984) and this difference may be related to a difference in strain rate being $10^{-3} \mathrm{~s}^{-1}$ and $10^{-2} \mathrm{~s}^{-1}$, respectively.

Figures 2-3 show that the flow stress in compression tends to decrease for strains above approximately 1.1. This flow softening has also been observed by Tome et al. (1984). Furthermore Figures 2-3 show that the flow stress increases with decreasing grain size both in compression and in tension. 


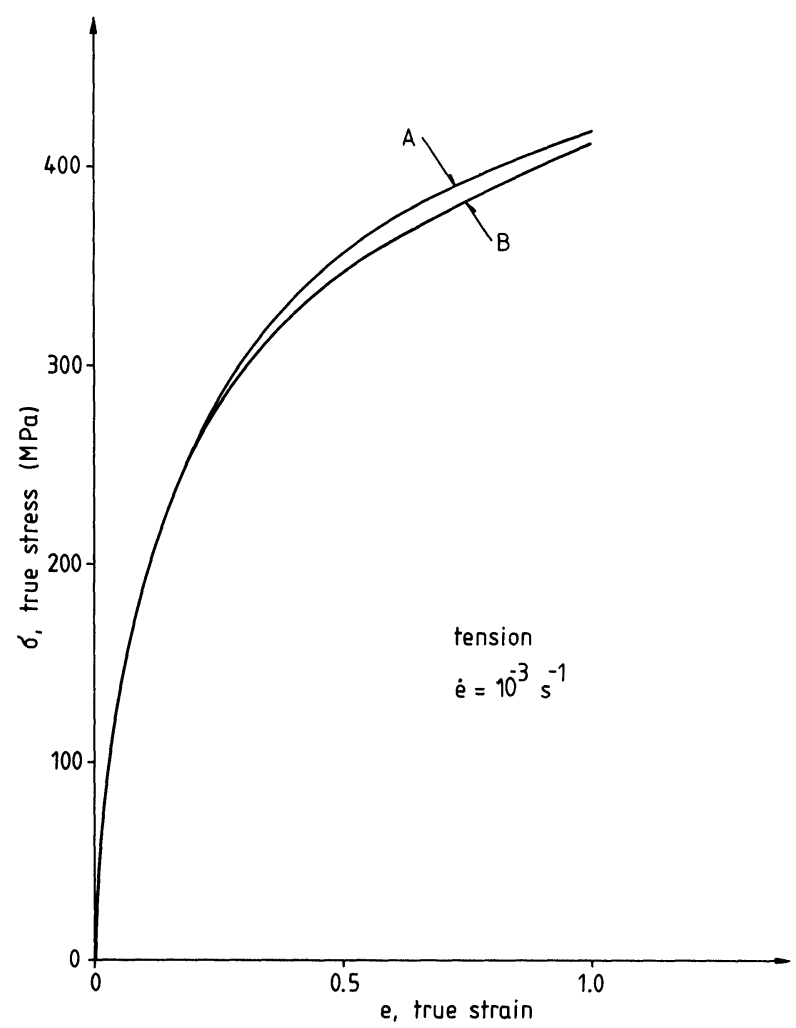

Figure 3 True stress-true strain curves in tension.

\section{B Deformation textures}

The textural development in compression is illustrated in Figure 4 for $A$ specimens. The overall trend is for strains below 1.4 that the $\langle 110\rangle$ component increases and that the $\langle 111\rangle$ and $\langle 100\rangle$ components decrease with increasing strain. The textures of the two specimens compressed to $e=2.176$ and $e=2.9$ are also shown in Figure 4. For these two specimens it is observed that the peak intensity of the $\langle 110\rangle$ component decreases and that the intensity of texture components lying in a band between $\langle 110\rangle$ and $\langle 115\rangle$ increases. 

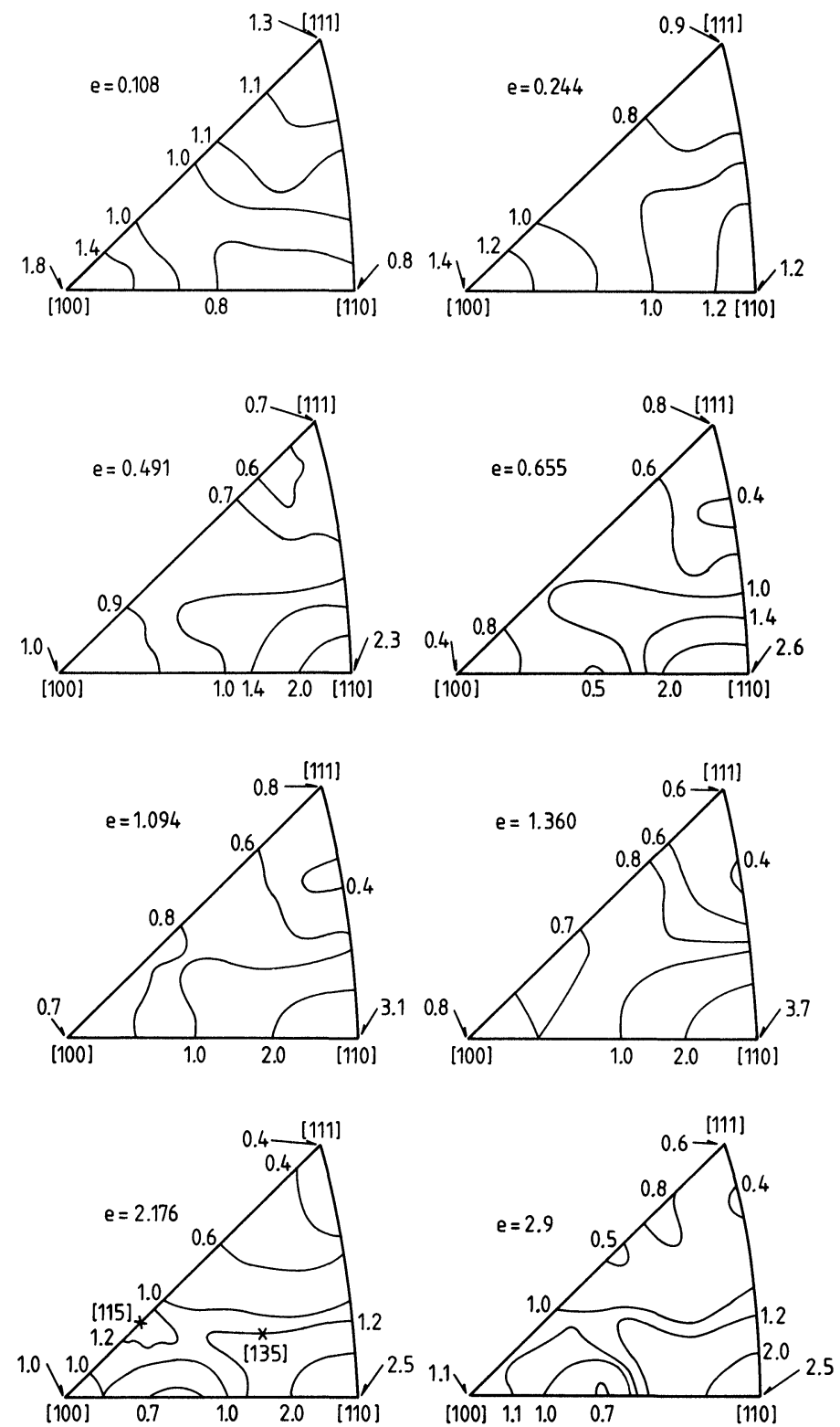

Figure 4 Inverse pole figures for $A$ specimens deformed in compression at different strains which are indicated on the pole figures. 
The volume concentration of the individual texture components have been calculated for specimens strained up to $e=1.36$. The concentration is calculated by integrating over an angle around the corresponding points in the inverse pole figure. Alternatively the concentration of several texture components in a large area in the

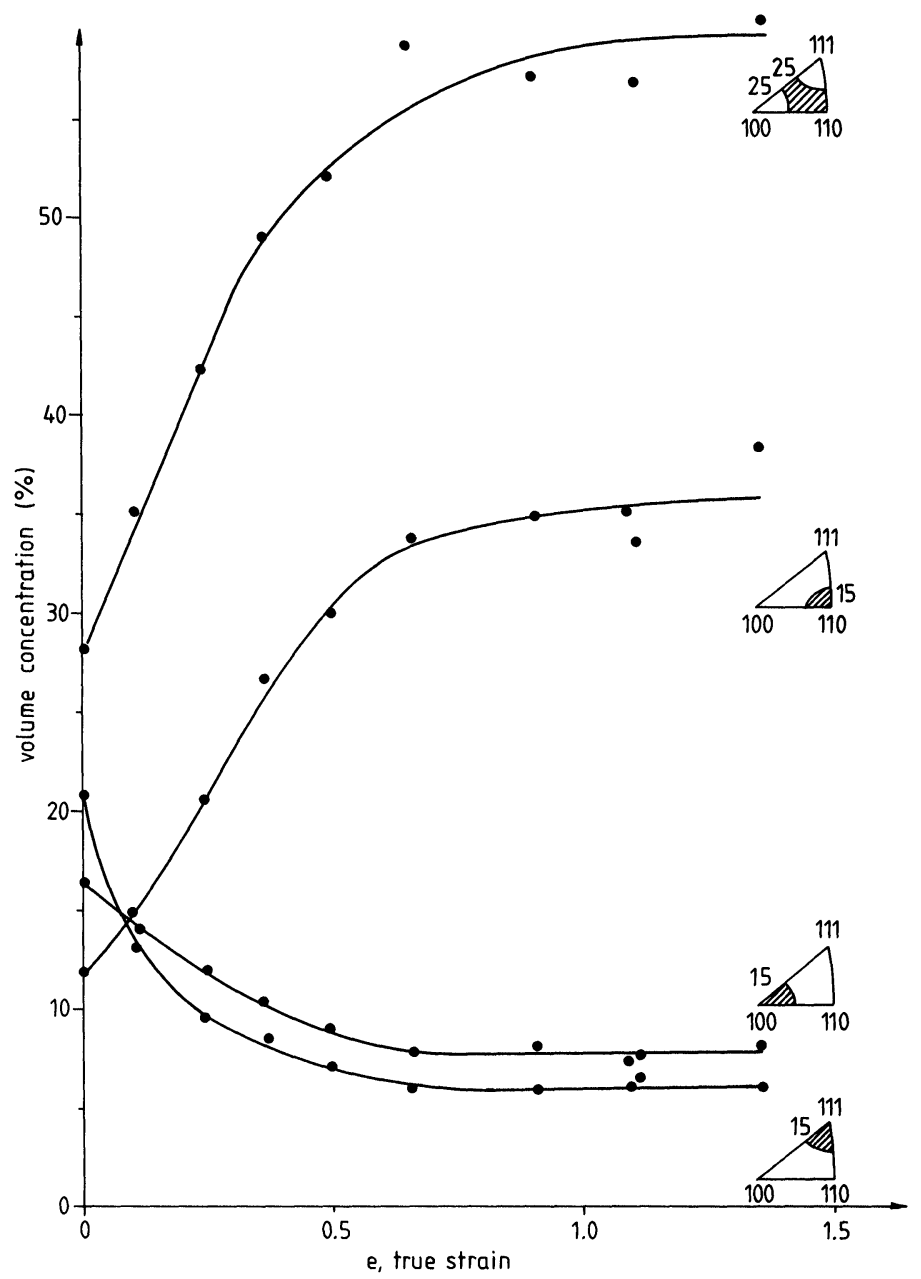

Figure 5 Volume concentrations of different texture components (hatched areas) for $A$ specimens deformed in compression. (The numbers on the stereographic triangles indicate the radii of integration). 
inverse pole figure is obtained by subtraction from $100 \%$ of the concentration of the remaining areas. For $A$ specimens the concentration of different texture components is given in Figure 5 as a function of strain.

The textural development in $A$ and $B$ specimens is compared in Figure 6 . To be noted in this figure is the difference in volume

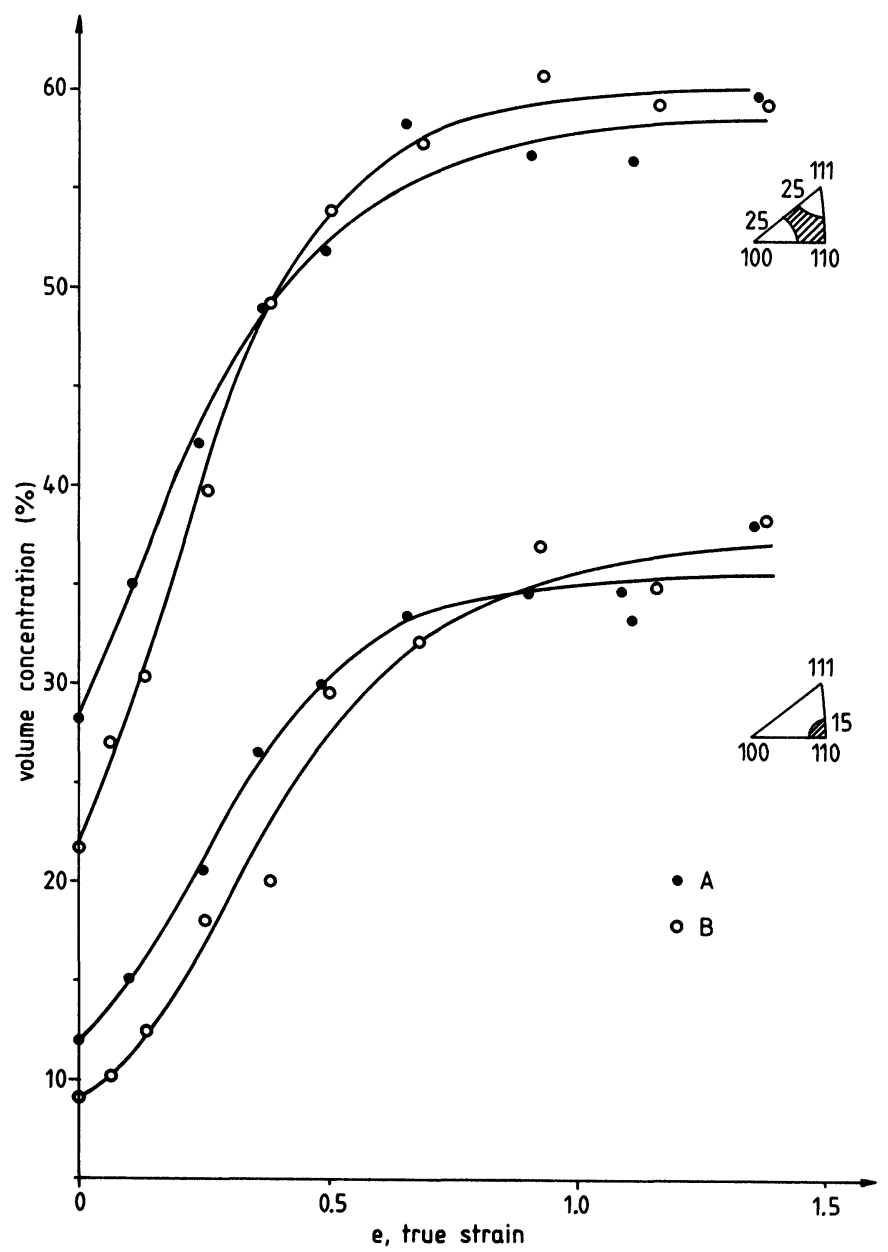

Figure 6 Volume concentrations of different texture components for $A$ and $B$ specimens deformed in compression. 
concentration for both texture components at small strains. However, this difference decreases with increasing strains.

The textural development in tension at strains below 0.3 is illustrated in Figure 7 for $A$ specimens of cylindrical geometry. The inverse pole figures show that the $\langle 111\rangle$ and $\langle 100\rangle$ components increase with increasing strain whereas the $\langle 110\rangle$ component decreases. These results are in good agreement with those obtained by Sam and Adams (1986) for tensile deformed copper having a grain size of approximately $21 \mu \mathrm{m}$. The textural development in $A$ and $B$ specimens is shown in Figure 8. A difference is observed at small strains between the two types of specimens and a difference can also be noted at larger strains in contrast to the results obtained by compression (Figure 6). The volume concentration of the individual texture components have been calculated for $A$ specimens deformed up to a strain of about 1.0 (see Figure 9). This
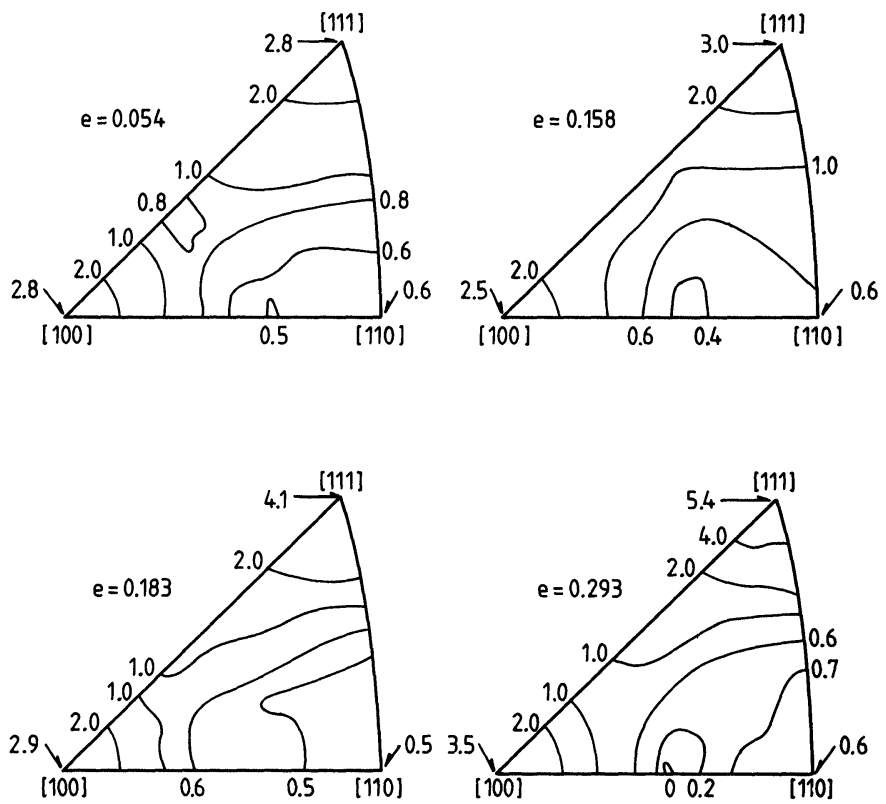

Figure 7 Inverse pole figures for $A$ specimens of cylindrical geometry deformed in tension at different strains which are indicated on the figure. 


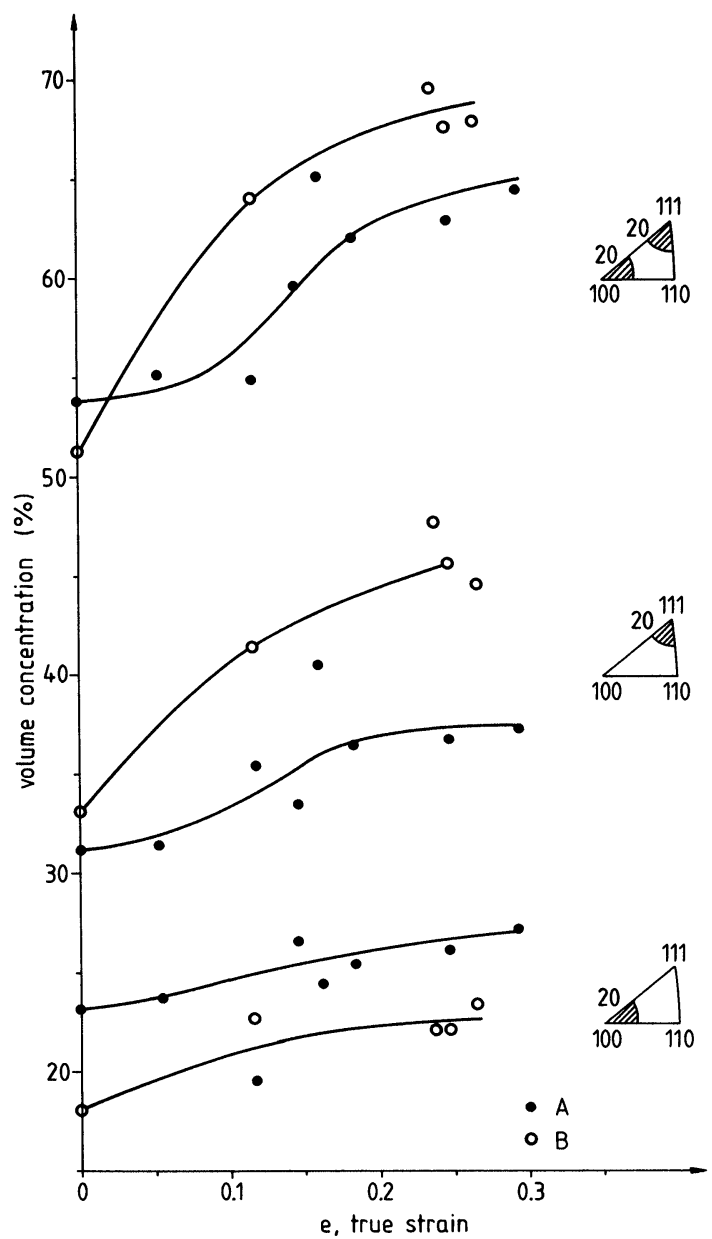

Figure 8 Volume concentrations of different texture components for $A$ and $B$ specimens of cylindrical geometry deformed in tension.

figure shows that the textural changes are small for strains larger than 0.5 .

\section{DISCUSSION}

The textural development observed during plastic deformation of polycrystals relates to the deformation mechanisms and to the 


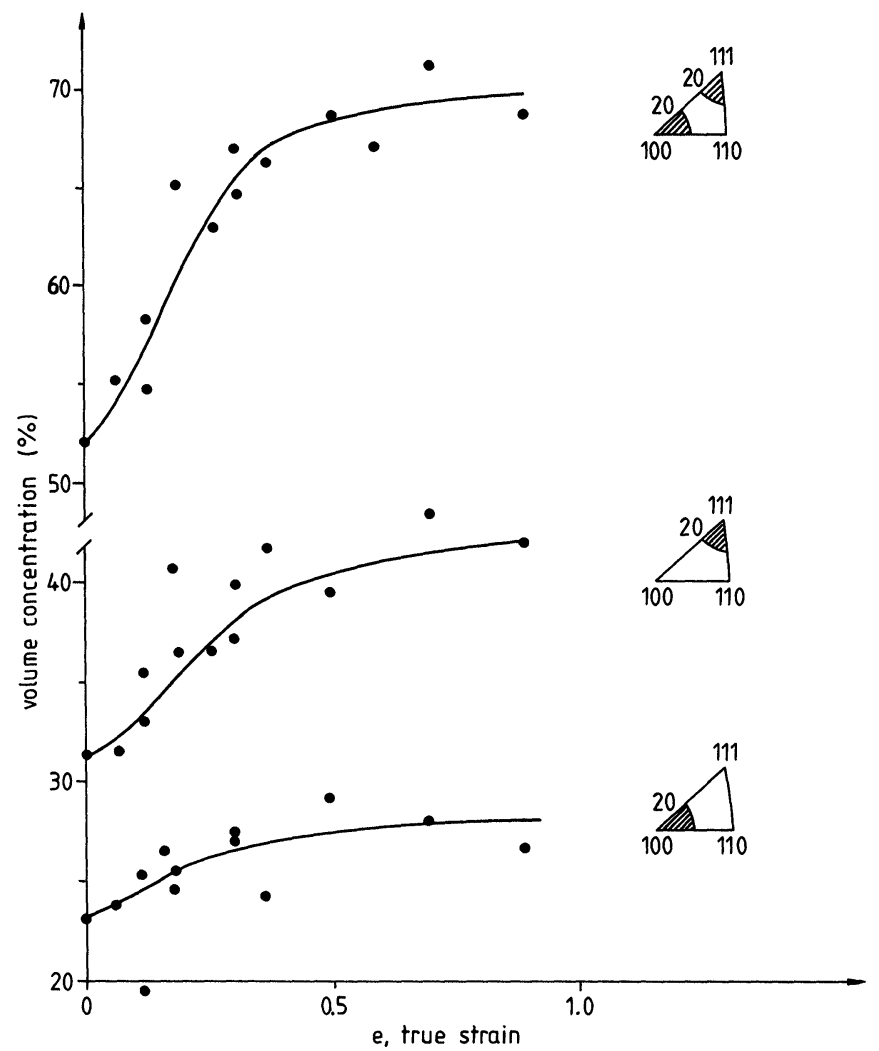

Figure 9 Volume concentrations of different texture components (hatched areas) for $\boldsymbol{A}$ specimens deformed in tension. (Cylinder- and hour glass shaped specimens).

stress-strain behaviour. These aspects will be dealt with in the following on the basis of models for the textural development.

\section{A Texture models}

A number of different models exists for the prediction of deformation textures (see introduction). Of these we shall consider the original Taylor model (Taylor, 1938) and a relaxed constraints (RC) model proposed for special grain shapes (Honeff and Mecking, 1978; Kocks and Canova, 1981; Tome et al., 1984). To maintain strain 
compatibility across the grain boundaries the Taylor model prescribes the operation of five independent slip systems in each grain whereas the RC-model prescribes a different number of slip systems (typically from 3 to 5) to be operative in various regions of the individual grains. This relaxation is based on an assumption of a change in grain shapes, e.g. to flat or elongated grains. In applying the Taylor model Tome et al. (1984) solve the problem of ambiguity with respect to grain rotations by calculating the resulting rotation as an average of the rotations for each combination of five systems.

A gradual change-over from the Taylor to the RC-model at increasing strain has been considered by Tome et al. (1984). Their model is based on a fcc polycrystal of 800 grains which are assumed to be equiaxed and randomly oriented. For deformation in compression and in tension Tome et al. (1984) assume that change-over to the RC approach starts at $e=0.46$, and that $90 \%$ of the grains are deforming in the RC-mode at $e=1.3$ and 2.0 in compression and tension, respectively. These texture calculations are reported as inverse pole figures and some examples are given in Figures 10 and 11 for compression and tension, respectively. In Figures 10a, 10b and 11a the predictions are based on the Taylor model whereas in Figures 10c and 11b the effect of a transition to the RC-model is included. In compression the transition results in a change in the predicted stable orientation from $\langle 110\rangle$ to a position between $\langle 110\rangle$ and $\langle 100\rangle$ (compare $10 \mathrm{~b}$ and 10c). In tension the effect of a transition has been found to be negligible, i.e. Figure $11 \mathrm{~b}$ represents a deformation texture typical for both models.

The predictions by Tome et al. (1984) shall in the following be compared with the experimental texture measurements.

\section{B Experimental observations and model predictions}

In comparing experimental results and those of model calculations it must be kept in mind that experimental conditions are not ideal. E.g. grains are not of equal size and completely equiaxed and the starting texture is normally not fully random. In spite of these limitations it seems that most of the experimental results are in broad agreement with model predictions although the rate of the texture development and the intensity of the final textures are overestimated in the models. These observations are common when 

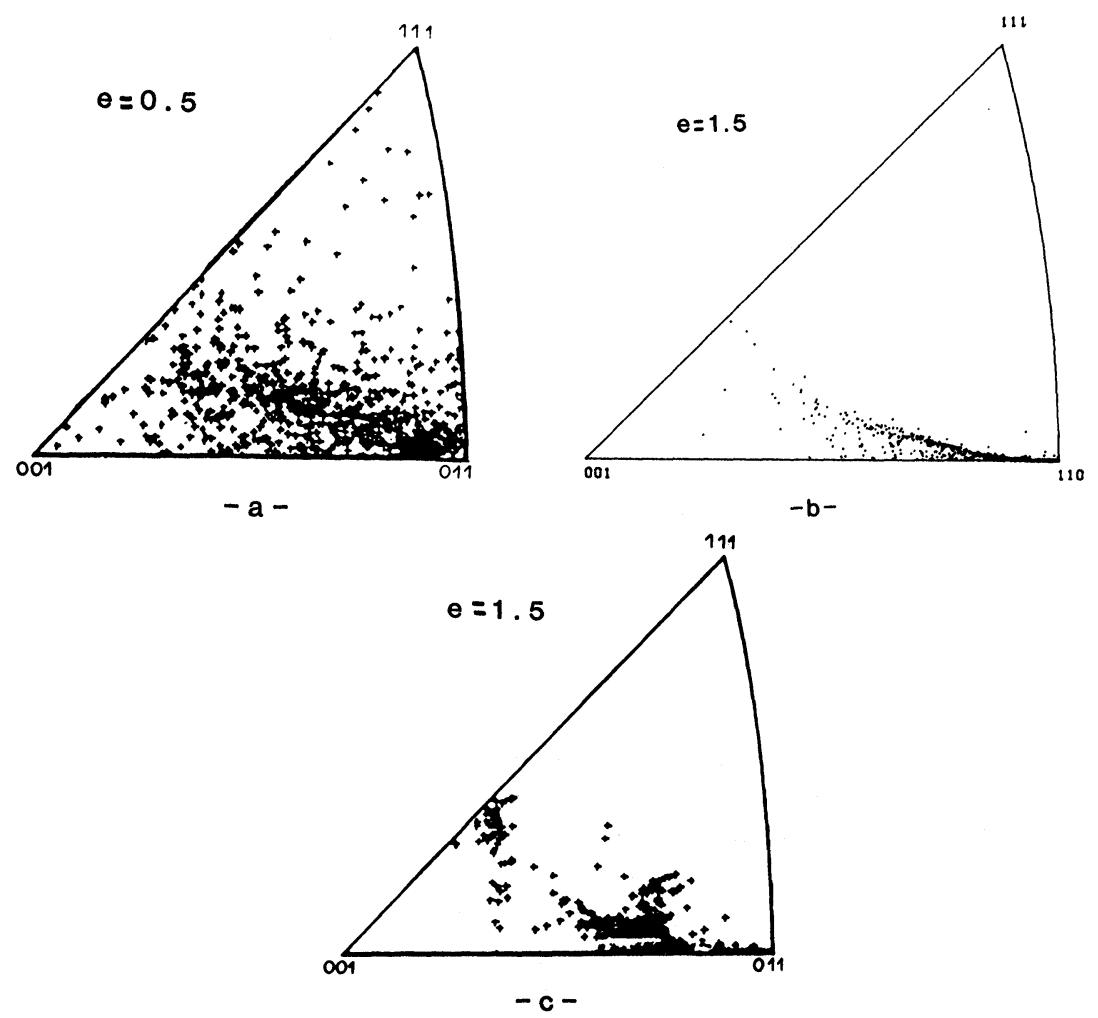

Figure 10 Model predictions. Inverse pole figures for specimens deformed in compression. Taylor-model (a, b) and RC-model (c). (a, c, Tome et al., 1984). (b, Bacroix, 1986).

comparing models and experiments, as discussed, for instance, by Aernoudt (1978).

Detailed comparison of experimental results and model predictions (Tome et al., 1984) shows a significant discrepancy in specimens deformed in compression at a strain of approximately 1.4. The experiments show a maximum concentration at the $\langle 110\rangle$ orientation (see Figure 4), which is in agreement with the predictions based on the Taylor model. Thus the RC-model suggested by Tome et al. (1984) (see Figure 10c) is not supported at the strain in question. The reason for this lack of agreement between experiment and model may be related to the assumption by Tome et al. (1984) 

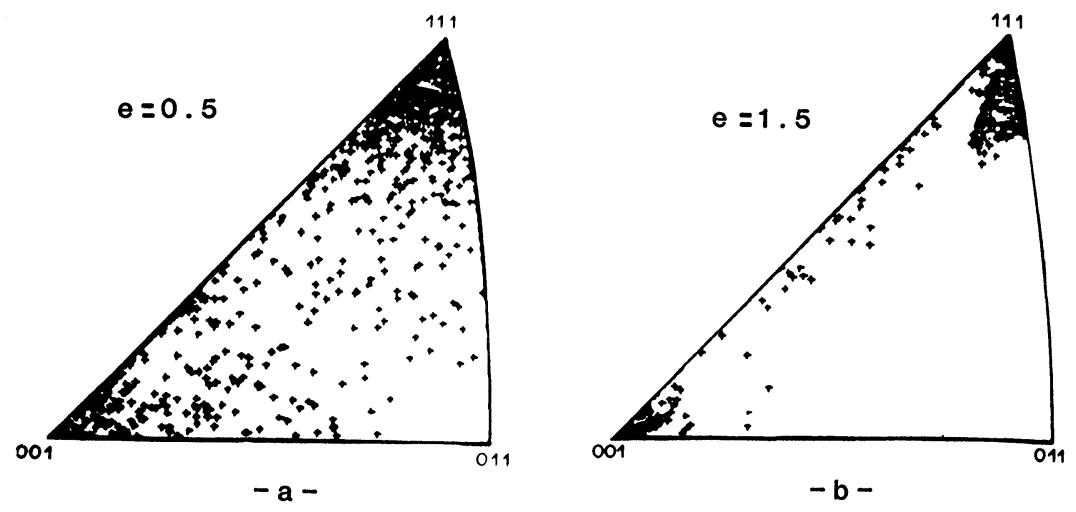

Figure 11 Model as in Figure 10 for specimens deformed in tension. Taylor-model (a) and RC-model (b). (Tome et al., 1984).

that the transition to the RC-model starts at $e=0.46$. This strain is not very large $\left(h / h_{0}=0.63\right)$ and it may be argued that the change in grain shape at this strain is too small to substantiate a transition. At larger strains $(e=2.176$ and $e=2.9)$ the concentration of the $\langle 110\rangle$ component decreases and components lying in a band between $\langle 110\rangle$ and $\langle 115\rangle$ increases (see Figure 4). This shift in texture development away from the $\langle 110\rangle$ texture brings the experimental results at large strains in broad agreement with the $\mathrm{RC}$ model predictions at lower strains (e.g. $e=1.5$ ). For strains of the order of $1.5-2.0\left(h / h_{0}=0.22-0.14\right)$ the grain flattening is pronounced and it appears quite reasonable that a transition to deformation in the RC-mode starts to take place at strains of this order.

For deformation in tension experimental results and model predictions are compared in Figure 12. The volume concentration of the sum of the $\langle 111\rangle$ and $\langle 100\rangle$ components is shown as a function of strain and relatively fair agreement is obtained considering the general discrepancy between experiments and models as concerns the rate of textural development and the intensity of the final texture.

The effect of grain size on the textural development has not been modelled. However, such an effect has been found earlier in copper (Leffers, 1974, Oztürk et al., 1981) and aluminium (Hansen et al., 1985) deformed in rolling. Generally it was observed that the 


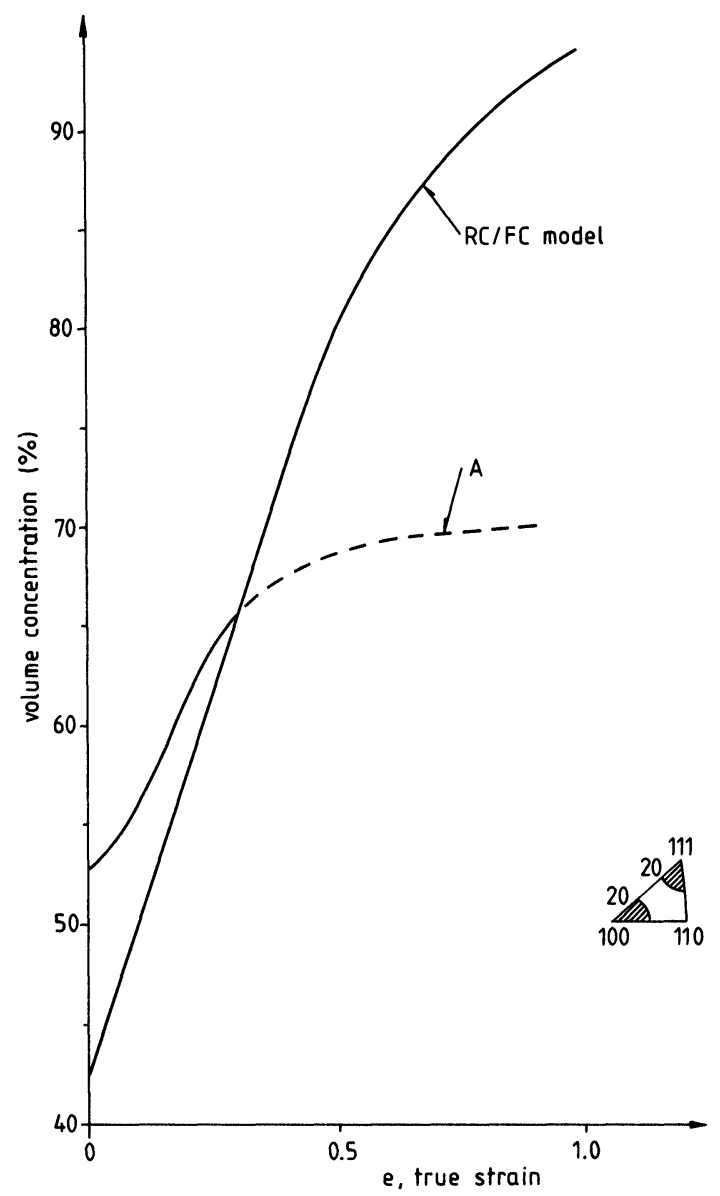

Figure 12 Textural development in tension. Model predictions based on the Taylor/RC-model (data, Bacroix, 1986) compared with experimental results for $A$ specimens (from Figure 9).

concentration of components developing during deformation as well as the rate of textural development may increase, when the grain size is decreased. However, in copper deformed in tension up to a strain of about 0.4 (Riegger, 1974) the textural development was almost identical in specimens having grain sizes of 10 and $300 \mu \mathrm{m}$. In the present experiment it appears that the textural development 
is only to a limited extent affected by the grain size. Also the starting texture may have an effect, which, however, appears to vanish with increasing strain when the deformation mode is compression. On the other hand a relatively small, initial difference in the concentration of the $\langle 111\rangle$ component between $A$ and $B$ specimens appear to increase with increasing strain in tension (see Figure 8). These results may indicate a rather complicated interplay between parameters such as starting texture, grain size, strain and deformation mode. A further examination of these parameters is required, e.g. by model calculations that introduce the effect of starting texture on the textural development (Leffers and Juul Jensen, 1986).

\section{Flow stress}

The initial texture and the textures developing during plastic deformation may affect the flow stress. This effect is normally introduced through the $M$-factor in the relation between the true tensile stress $(\sigma)$ and the critical resolved shear stress $(\tau)$

$$
\sigma=M \tau
$$

$\tau$ relates to the hardening of the individual grains and the $M$ factor expresses the effects both of interactions between grains and of grain orientations. The $M$-factor depends on the deformation mode as well as the number of slip systems required for strain accommodation across grain boundaries.

Deformation modelling according to the Taylor approach leads to an increase in $M$ with increasing strain both in compression and in tension (Gil Sevillano et al., 1980, Tome et al., 1984). The present findings show a qualitative agreement between experimental textures (for compression up to $e=1.36$ ) and those predicted on the basis of the Taylor model. Thus experimental $M$-values can be derived from $M$-values calculated on the basis of the Taylor model for different orientations in the stereographic triangle (Hosford and Backofen, 1962). This triangle is divided into 100 equivalent areas and the $M$-factor for each area is derived. On the basis of these values and the volume concentration of grains having orientation within the same areas the experimental $M$-values are calculated and summed up for the whole triangle. The $M$-values for both the fine 

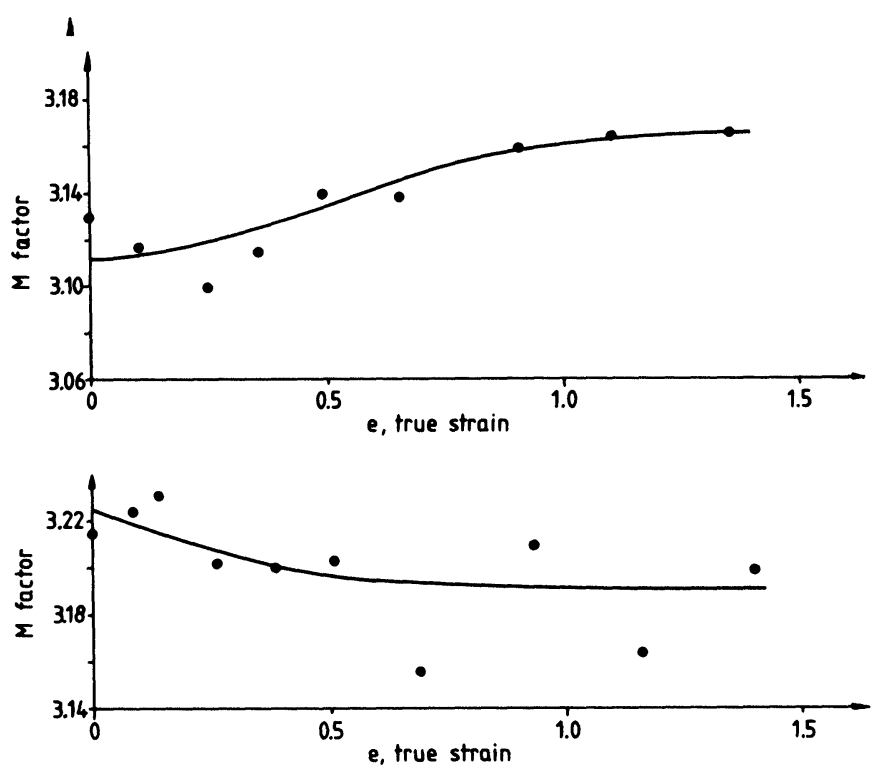

Figure 13 Experimental $M$-factors calculated on the basis of the Taylor-model for $A$ and $B$ specimens deformed in compression. (Top curve $A$ specimen, bottom curve $B$ specimen).

and the coarse grained starting material are shown in Figures 13 and 14 for compression and tension, respectively. The changes in the experimental $M$-values with increasing strain are small and much less pronounced than these obtained by model calculations (Gil Sevillano et al., 1980, Tome et al., 1984). This finding agrees with the observation discussed above that model predictions tend to over-estimate the concentration of the components of the deformation texture.

A change in $M$ as a function of strain leads to hardening or softening for purely geometrical reasons. However such an effect is considered to be insignificant for strains below 1.4 due to the small variation in $M$ with increasing strain. Therefore the flow softening observed in compression at strains above 1.1 cannot be explained on basis of the strain dependency of $M$ (see Figure 13). The problem of flow softening has been discussed by Tome et al. (1984) who explained this phenomenon as a result of a transition from the 

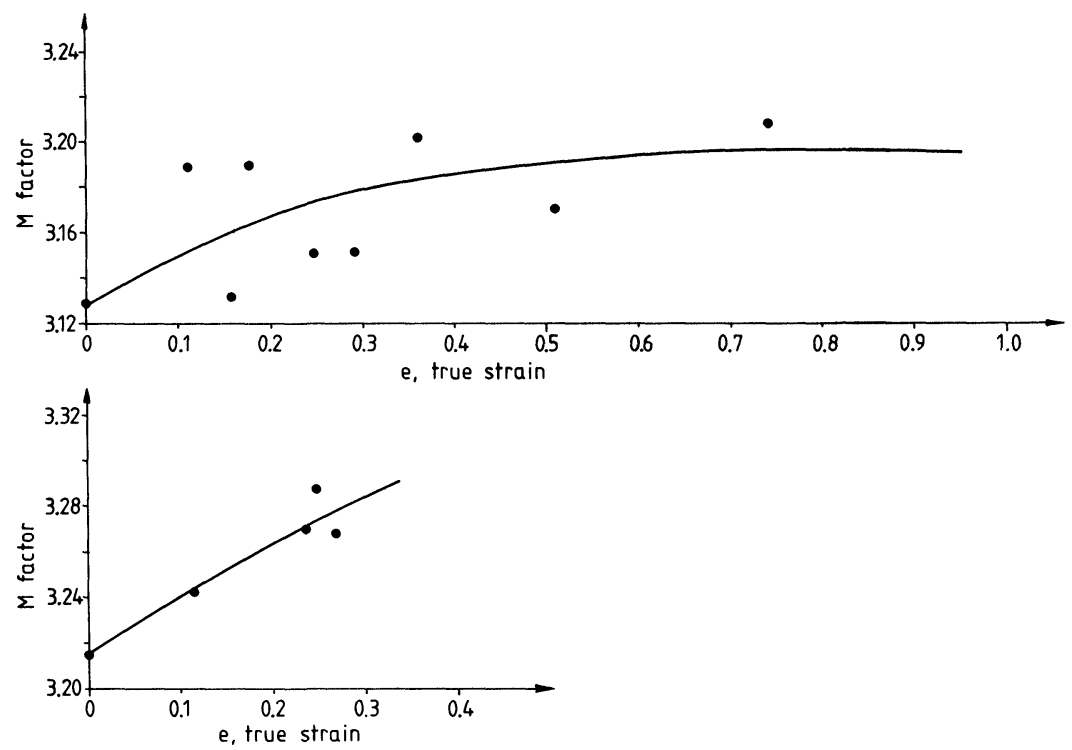

Figure 14 Experimental $M$-factors calculated on basis of the Taylor-model for $A$ and $B$ specimens deformed in tension. (Top curve $A$ specimen, bottom curve $B$ specimen).

Taylor-model to the RC-model causing a reduction in $\boldsymbol{M}$. However, the present experiments indicate that such a transition does not take place at strains as low as 1.1 and other explanations for flow softening must be sought. Tome et al. (1984) suggested that dynamic recrystallization, formation of deformation twins and of shear bands may cause flow softening, but they could not find any metallographical evidence for such processes, which therefore were ruled out. Alternatively, it has been suggested by Hosford (1964) that once a $\langle 110\rangle$ fiber texture has formed (as in the present case) the slip systems are oriented in such a way that further slip is by plane strain. This type of deformation requires less plastic work than axisymmetric deformation (Hosford, 1964) and may therefore be a cause of softening. In the plain strain mode compatibility between individual grains can be obtained only if they bend about one another, the so-called curling. This phenomena was observed by Hosford (1964) in pure aluminium $(99.999 \%)$ compressed in the range $73-89 \%(e=1.3-2.2)$. 


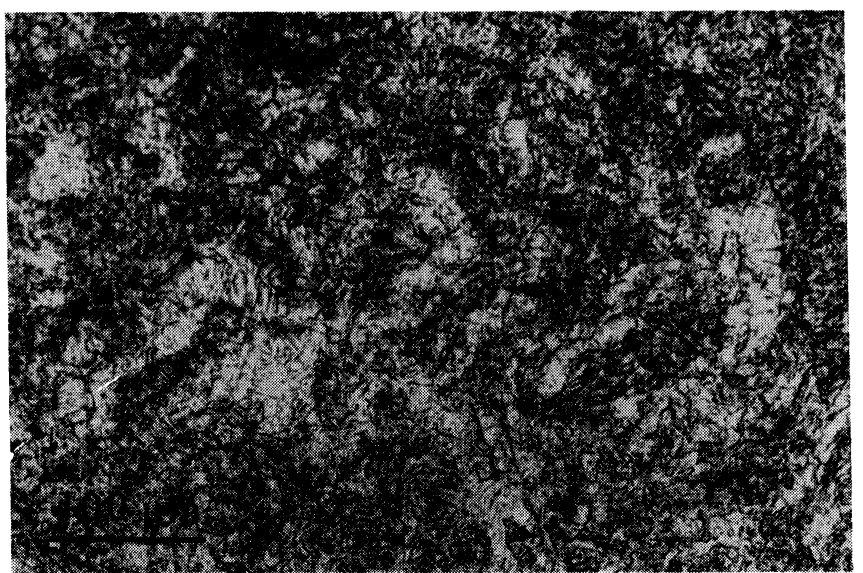

Figure 15 Optical micrograph showing a cross section of an $A$ specimen after compression to $e=2.9$.

A metallographical examination of specimens compressed to a strain of 2.9 (see Figure 15) demonstrates the same structure as observed by Hosford (1964) thus curling also occurs in the present specimens. Thus it is tentatively suggested that the flow softening observed at strains above 1.1 may be due to the formation of a relatively strong $\langle 110\rangle$ texture giving rise to a change away from axisymmetric deformation. How such a change will affect the textural development has not been investigated. However, it can be argued that the textural changes observed in compression at large strains may have their cause either in grain flattening (suggested in the RC-model) or in grain curling or in a combination of the two mechanisms.

The effect of the grain size on the $M$-factors as a function of strain appears to be relatively small (see Figures 13, 14). However, for tension, the $M$-factor in the $B$-specimens having the larger grain size appears to increase somewhat faster with strain than the $M$-factor for the $A$-specimens. The $M$-factor also starts at a higher value in the $B$-specimens, thus a larger geometrical strengthening must be expected in these specimens. This observation may relate to the findings that coarse grained copper specimens (often having a relatively strong initial $\langle 111\rangle$ texture) at increasing strain may 
become as strong or stronger than fine-grained specimens (Hansen, 1985). This problem of texture effects on the flow stress of copper having different grain sizes will be dealt with in a forthcoming paper.

\section{CONCLUSIONS}

- The textural development in copper $(99.999 \%)$ in compression $(e<1.4)$ and in tension $(e<1.0)$ is in qualitative agreement with predictions based on the Taylor-model. However, the rate of textural changes and the concentration of the textural components are largely overestimated in the model. For large strains $(e=1.4-2.9)$ in compression the textural development is in broad accordance with RC-model predictions.

- The effect of grain size (23 and $125 \mu \mathrm{m})$ on the textural development is small both in compression $(e<1.4)$ and in tension $(e<0.3)$.

- The textural changes observed do not affect the $M$-factor to a large extent in the strain range where the Taylor-model is prevalent.

- Flow softening is observed in compression at $e>1.1$. This phenomenon is related to the formation of a relatively strong $\langle 110\rangle$ texture, which may cause a transition from axisymmetric to plane strain flow.

\section{Acknowledgement}

We thank T. Leffers for helpful discussions, B. Bacroix and J. J. Jonas for providing the data included in Figures 10-12, and G. W. Lauritsen, H. Nilsson, P. Nielsen, E. D. Ravn and E. Sørensen for assistance with the experimental work and with the preparation of the manuscript.

\section{References}

Aernoudt, E. (1978). Proc. 5th Int. Conf. on Textures of Materials (ICOTOM-5) (edited by G. Gottstein and K. Lücke), 45-65, Springer, Berlin.

Bacroix, B. (1986). PhD Thesis to be published. 
Bridgman, P. W. (1952). Studies in Large Plastic Flow and Fracture. McGraw-Hill, New York.

Chin, G. Y., Mammel, W. L. and Dolan, M. T. (1967). Trans. Met. Soc. AIME, 239, 1111-1112, 1854-1855.

Gil Sevillano, J., van Houtte, P. and Aernoudt, E. (1980). Prog. Mat. Sci., 25, 69-412.

Hansen, N., Bay, B., Juul Jensen, D. and Leffers, T. (1985). Proc. 7th Int. Conf. on the Strength of Metals and Alloys, (edited by H. J. McQueen, J.-P. Bailon, J. I. Dickson, J. J. Jonas and M. G. Akben), 317-322, Pergamon Press, Oxford.

Hansen, N. (1985). Metall. Trans. A, 16A, 2167-2190.

Honeff, H. and Mecking, H. (1978). Proc. 5th Int. Conf. on Textures of Materials (ICOTOM-5) (edited by G. Gottstein and K. Lücke), 265-275, Springer, Berlin.

Hosford, W. F. (1964). Trans. Met. Soc. AIME, 230, 12-15.

Hosford, W. F. and Backofen, W. A. (1962). Strength and Plasticity of Textured Metals. Ninth Sagamore Army Materials Research Conference Proceedings, New York.

Juul Jensen, D. and Kjems, J. K. (1983). Textures and Microstructures, 5, 239-251.

Juul Jensen, D., Hansen, N., Kjems, J. K. and Leffers, T. (1984). Proc. Sth Ris $\emptyset$ Int. Symp., Microstructural Characterization of Materials by Non-Microscopical Techniques (edited by N. Hessel Andersen, M. Eldrup, N. Hansen, D. Juul Jensen, T. Leffers, H. Lilholt, O. B. Pedersen and B. N. Singh), 325-332, Ris $\varnothing$ Nat. Lab., Roskilde, Denmark.

Kallend, J. S. and Davies, G. J. (1972). Phil. Mag., 25, 471-489.

Kocks, U. F. and Canova, G. R. (1981). Proc. 2nd Ris $\varnothing$ Int. Symp., Deformation of Polycrystals: Mechanisms and Microstructures (edited by N. Hansen, A. Horsewell, T. Leffers and H. Lilholt), 35-44, Ris $\emptyset$ Nat. Lab., Roskilde, Denmark.

Leffers, T. (1974). Metall. Trans., 5, 2110-2112.

Leffers, T. (1968). Risø Report No. 184, Danish Atomic Energy Commission.

Leffers, T. and Juul Jensen, D. (1986). This volume.

Oztürk, T., Kallend, J. S. and Davies, G. J. (1981). Proc. 6th Int. Conf. on Textures of Materials (ICOTOM-6), 507-518, The Iron and Steel Institute of Japan.

Riegger, S. (1974). Dissertation, University of Karlsruhe.

Sam, D. D. and Adams, B. L. (1986). Metall. Trans. A., 17A, 513-517.

Taylor, G. I. (1938). J. Inst. Met., 62, 307-324.

Taylor, G. I. and Quinney, H. (1934). Proc. Roy. Soc. Lond. 143, 307-326.

Tome, C., Canova, G. R., Kocks, U. F., Christodoulou, N. and Jonas, J. J. (1984). Acta Metall. 32, 1637-1653. 